\title{
EQUIVALENCE OF THE THIN-GRATING DECOMPOSITION AND COUPLED-WAVE ANALYSIS OF THICK HOLOGRAHIC GRATINGS
}

\author{
R. ALFERNESS \\ Electro-Optics Laboratory, Department of Electrical and Computer Engineering, \\ The University of Michigan, Ann Arbor, Michigan 48104, USA
}

Received 10 June 1975

The method of thin grating decomposition, which is derived entirely from thin-grating diffraction theory, is shown analytically to be equivalent to the coupled-wave solution of Maxwell's equations for a thick sinusoidal grating.

Recently a new and physically intuitive method for analyzing wave propagation in thick holographic gratings was described [1]. The method proceeds by decomposing the thick material into a series of thin slabs, each of which acts simply as a thin grating. For a readout plane wave of arbitrary spatial frequency, the total amplitude of the zero and diffracted orders is determined by computing the successive effect of each thin grating upon the propagating waves. The effect of each thin grating is described by a coupling matrix which is determined solely from thin grating theory. The $i, j$ element of the coupling matrix gives the amplitude and phase shift effected by the thin grating when it diffracts the $j$ th plane wave into the $i$ th one. Because the output plane waves from one thin grating are incident upon the subsequent one, the total effect is found by successively multiplying each of the coupling matrices. In general, several diffracted orders may be significant and the necessary matrix multiplication must be performed by a digital computer. The advantages of the method are its intuitive simplicity and general applicability. For example, the method has been employed to study the strong coupling which occurs between two gratings with common or nearly common Bragg angles recorded in the same emulsion [2].

Previously we have shown numerically the equivalence between the results of the above theory and Kogelnik's [3] coupled-wave solution of Maxwell's equations for a thick grating for which his assumptions are valid [1]. In this note we show this equivalence analytically.

We consider a pure phase grating const ructed holographically as shown in fig. 1 . We assume a sinusoidal index modulation and there is no variation along the $y$ direction. The readout beam is in the $x, z$ plane and is polarized along the fringe lines. The grating is assumed to have a sufficiently high $Q$ factor [3] such that only the zero and one diffracted order are significant for

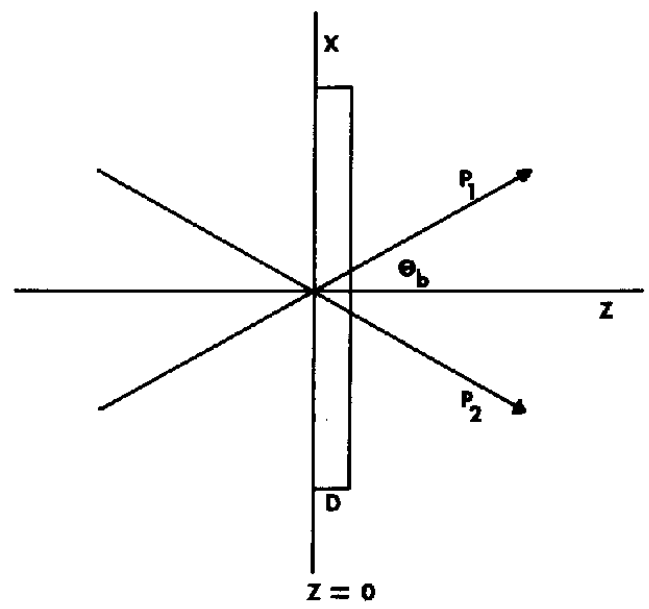

Fig. 1. Holographically constructed grating. Plane waves $P_{1}$ and $P_{2}$ interfere to produce a sinusoidal grating of spatial frequency $f$ throughout the volume of the emulsion. The propagation vector of $P_{1(2)}$ makes an angle $\theta_{b}\left(-\theta_{b}\right)$ with respect to the $z$ axis. The emulsion thickness is $D$. 
readout about the 1st order Bragg angle. Because only two orders are important, the coupling matrix for the $n$th thin grating [1] can be truncated to a $2 \times 2$

matrix,

${ }_{n} H=\left(\begin{array}{cc}\mathrm{e}^{\mathrm{j} \alpha_{0}} J_{0}\left(b_{0}\right) & \mathrm{je} \mathrm{e}^{\mathrm{j} \alpha_{1}} J_{1}\left(b_{1}\right) \mathrm{e}^{\mathrm{j} \delta(n)} \\ \mathrm{j \textrm {j }} \mathrm{e}^{\mathrm{j} \alpha_{0}} J_{-1}\left(b_{0}\right) \mathrm{e}^{-\mathrm{j} \delta} & \mathrm{e}^{\mathrm{j} \alpha_{1}} J_{0}\left(b_{1}\right)\end{array}\right)$,

where

$b_{l}=\frac{2 \pi \Delta z n_{1}}{\lambda \sqrt{1-\left(\lambda / n_{0}\right)^{2} f_{l}^{2}}}$

$\alpha_{l}=\frac{2 \pi \Delta z n_{0}}{\lambda} \sqrt{1-\left(\lambda / n_{0}\right)^{2} f_{l}^{2}}, \quad l=0,1$,

$\delta=-2 \pi n f \tan \phi$.

$f_{0}=f_{i}$ is the spatial frequency of the readout wave, $f_{1}=f_{i}-f$ is the spatial frequency of the diffracted order, $f$ is the spatial frequency of the grating in any $z=$ const. plane, $\lambda$ is the free space wavelength of the readout wave, $\phi$ is the slope of the fringes with respect to the $z$ axis, $n_{1}$ is the index modulation of the grating, $n_{0}$ is the bulk index of the emulsion and $\Delta z$ is the thickness of the thin grating slab. For simplicity we assume that the fringe planes are perpendicular to the emulsion, $\phi=0$. Therefore, the phase factors of cq. (1) due to fringe slope vanish and all the coupling matrices are identical. Also, for typical values of index modulation and because we will take $\Delta z$ arbitrarily small, we can approximate the Bessel functions by their first order approximations, $J_{0}(x) \simeq 1, J_{1}(x) \simeq \frac{1}{2} x$ for $x \ll 1$. Furthermore, since $f_{l}=\left(n_{0} \sin \theta_{l}\right) / \lambda$ we have $\sqrt{1-\left(\lambda / n_{0}\right)^{2} f_{l}^{2}}=\cos \theta_{l}$. Note that the correspondence to Kogelnik's notation for the subscripts of the readout and diffracted waves is $0 \rightarrow \mathrm{r}$ and $1 \rightarrow \mathrm{s}$. The coupling matrix for each thin grating can therefore be written as

$$
\begin{aligned}
& H=\exp \left(\mathrm{j} \frac{2 \pi n_{0}}{\lambda} \Delta z \cos \theta_{1}\right) \\
& \times\left(\begin{array}{cc}
1 & \mathrm{j} \frac{\gamma}{\cos \theta_{1}} \mathrm{e}^{\mathrm{j} 2 \Phi} \\
\mathrm{j} \frac{\gamma}{\cos \theta_{0}} & \mathrm{e}^{\mathrm{j} 2 \Phi}
\end{array}\right),
\end{aligned}
$$

where $\gamma=\pi \Delta z n_{1} / \lambda, 2 \Phi=(2 \pi / \lambda) n_{0} \Delta z$ $\times\left(\cos \theta_{1}-\cos \theta_{0}\right)$ and we have also used, $J_{-1}(x)=$ $-J_{1}(x)$. All angles are internal.

If the amplitudes of the zero and first order at the $n$th thin grating are expressed by the elements of a column vector

$A(n)=\left(\begin{array}{c}A_{0} \\ A_{1}\end{array}\right)_{n}$,

then the value of $\boldsymbol{A}$ at the exit plane of the emulsion, $z=D$ is [1]

$\boldsymbol{A}(N)=H^{N} \boldsymbol{A}(0)$,

where $N=D / \Delta z$ and

$A(0)=\left(\begin{array}{l}1 \\ 0\end{array}\right)$

Here we analytically perform the matrix multiplication of eq. (4) in the limit $N \rightarrow \infty, \Delta z \rightarrow 0$ such that $N \Delta z=D$.

Before performing the above matrix multiplication, we wish to simplify and examine the significance of the parameter $\Phi$. The direction $\operatorname{cosines} \cos \theta_{0}$ and $\cos \theta_{1}$ are related through the grating equation $f_{1}=$ $f_{0}-f$. We wish to determine the change in diffraction efficiency that results when the readout angle or wavelength differ from their construction values. Therefore we write,

$\theta_{0}=\theta_{\mathrm{b}}+\Delta \theta, \quad \lambda=\lambda_{\mathrm{c}}+\Delta \lambda$,

where $\Delta \theta \ll 1, \Delta \lambda / \lambda_{c} \ll 1, \lambda_{c}$ is the wavelength of the construction beams and $\theta_{\mathbf{b}}$ is the angle of each of the construction beams with respect to the $z$ axis. Thus, it is the Bragg angle that corresponds to $\lambda_{c}$. In terms of the construction parameters the spatial frequency of the grating (fig. 1$)$ is $\left(2 n_{0} / \lambda_{\mathrm{c}}\right) \sin \theta_{\mathrm{b}}=f$. From the thin grating equation above and trigonometric expansions, we find

$\cos \theta_{0}=\cos \theta_{\mathrm{b}}-\Delta \theta \sin \theta_{\mathrm{b}}$,

$\cos \theta_{1}=\cos \theta_{\mathrm{b}}+\Delta \theta \sin \theta_{\mathrm{b}}-\tan \theta_{\mathrm{b}}\left(\Delta \lambda f / n_{0}\right)$,

where we have dropped terms of second order in $\Delta \theta$ and $\Delta \lambda$. It is interesting to note from eq. (7) that at the Bragg angle and for $\Delta \lambda=0$, the readout and diffracted waves have the same inclination with respect to the fringe planes, i.e. the reflection law is followed. 
Indeed, Bragg diffraction is frequently viewed as reflection off the fringe planes. In general, however, the reflection law is not obeyed. Substituting the expressions for the direction cosines of eq. (7) into the expression for $\Phi$ we find,

$\Phi=\frac{2 \pi}{\lambda_{\mathrm{c}}} \Delta z n_{0} \sin \theta_{\mathbf{b}}\left(\Delta \theta-\frac{\Delta \lambda f}{2 n_{0} \cos \theta_{\mathrm{b}}}\right)$.

As shown by the coupling matrix of eq. (2), $2 \Phi$ represents a relative phase shift between the two waves that is produced by each thin grating when the reconstruction wavelength or angle or both differ from their construction values. It is this phase shift that causes the decrease in diffraction efficiency for readout waves incident off the Bragg angle. $\Phi$ corresponds to the dephasing parameter of the coupled-wave theory as we will show below. The term in parenthesis in eq. (8) shows the relative effect of readout angle change compared to wavelength change from their construction values in causing this phase shift.

The matrix multiplication of eq. (4) is greatly simplified when $H$ is represented in a basis such that it is diagonal. The diagonal elements are just the eigenvalues of $H$ and the basis vectors are the associated eigenvectors. The eigenvalues of $H$ can easily be shown to be,

$\psi_{1,2}=\exp (\mathrm{j} \Phi) \exp \left[ \pm \mathrm{j}\left(\Phi^{2}+\gamma^{2} / c_{0} c_{1}\right)^{1 / 2}\right]$,

where we have used the limit $\Delta z \rightarrow 0$ to set $\sin ^{2} \Phi=\Phi^{2}$. Also $c_{0} \equiv \cos \theta_{0}$ and $c_{1} \equiv \cos \theta_{1}$. The associated eigenvectors $E_{1}, E_{2}$ are then

$\left(\begin{array}{c}E_{1} \\ E_{2}\end{array}\right)=\left(\begin{array}{ll}1 & a_{1} \\ 1 & a_{2}\end{array}\right)\left(\begin{array}{l}A_{0} \\ A_{1}\end{array}\right)$,

where $a_{i}=\mathrm{j}\left(c_{1} / \gamma\right)\left(1-\psi_{i}\right) \mathrm{e}^{-\mathrm{j} 2 \Phi}$. From eq. (10) and the initial condition on $\boldsymbol{A}$ [eq. (5)], we find the initial condition in the $E$ basis, $E(0)=\left(\begin{array}{l}1 \\ 1\end{array}\right)$. Therefore in the diagonal representation,

$E(N)=\left(\begin{array}{cc}\psi_{1}^{N} & \\ & \psi_{2}^{N}\end{array}\right)\left(\begin{array}{l}1 \\ 1\end{array}\right)=\left(\begin{array}{l}\psi_{1}^{N} \\ \psi_{2}^{N}\end{array}\right)$.

We next use the inverse of the transformation of eq. (10) to transform back to the original representation to find the amplitude of the diffracted order,

$A_{1}(N)=\frac{1}{a_{2}-a_{1}}\left(\psi_{2}^{N} \quad \psi_{1}^{N}\right)$.
The intensity of the diffracted order is therefore,

$$
\left|A_{1}(N)\right|^{2}=\left(\frac{\gamma}{c_{1}}\right)^{2} \frac{\sin ^{2}\left[N\left(\Phi^{2}+\gamma^{2} / c_{0} c_{1}\right)^{1 / 2}\right]}{\Phi^{2}+\gamma^{2} / c_{0} c_{1}}
$$

Kogelnik [3] defines diffraction efficiency (for a unit input amplitude $\left.A_{0}\right)$ to be $\left(\left|\cos \theta_{1}\right| / \cos \theta_{0}\right)\left|A_{1}\right|^{2}$. There fore we find from the thin grating decomposition approach that the diffraction efficiency is,

$$
\text { D.E. }(N)=\frac{\sin ^{2}\left[N^{2} \Phi^{2}+N^{2} \gamma^{2} / c_{0} c_{1}\right]^{1 / 2}}{1+\Phi^{2} /\left(\gamma^{2} / c_{0} c_{1}\right)}
$$

To compare this result with the coupled-wave solution of Maxwell's equations for the thick grating, we make the correspondence between the parameters above and those of Kogelnik's expression. Because $N \Delta z=D$,

$\frac{N^{2} \gamma^{2}}{c_{0} c_{1}}=\frac{\pi^{2} D^{2} n_{1}^{2}}{\lambda^{2} c_{0} c_{1}}$

which is $\nu^{2}$ in Kogelnik's notation. Furthermore because $\cos \theta_{1} \simeq \cos \theta_{\mathrm{b}}$ we can write,

$$
\begin{aligned}
N^{2} \Phi^{2} & =\left\{\frac{2 \pi D n_{0} \sin \theta_{\mathrm{b}}}{\lambda_{\mathrm{c}}}\left(\Delta \theta-\frac{f \Delta \lambda}{2 n_{0} \cos \theta_{\mathrm{b}}}\right)\right\}^{2} \\
& =\left\{\frac{K D \Delta \theta \cos \theta_{\mathrm{b}}}{2 \cos \theta_{1}}-\frac{K^{2} D \Delta \lambda}{8 \pi n_{0} \cos \theta_{1}}\right)^{2},
\end{aligned}
$$

where $K=2 \pi f$ and we have used $f=\left(2 n_{0} \sin \theta_{\mathrm{b}}\right) / \lambda_{\mathrm{c}}$. Therefore $N^{2} \Phi^{2}$ is $\xi^{2}$ in Kogelnik's notation. Thus eq. (14) can be written as

D.E. $=\frac{\sin ^{2}\left(\nu^{2}+\xi^{2}\right)^{1 / 2}}{1+\xi^{2} / v^{2}}$.

which is identical to that obtained by Kogelnik (eq. (43), ref. [3]).

We have shown analytically the equivalence between the method of thin grating decomposition and Kogelnik's coupled-wave solution for wave propagation in a thick sinusoidal grating for which the assumptions of the latter theory are valid. The method of thin grating decomposition is derived completely from thin grating theory and does not require explicitly solving Maxwell s equations for a thick material. It therefore has the advantage of being intuitively physical and as shown here also gives accurate results. 
The method is also quite general in that higher orders can be included and multiple gratings in the same emulsion can be analyzed.

The author wishes to thank Professor E.N. Leith for helpful discussions during the course of this work. The generous support of the National Science Foundation (Grant GK-42361) is also greatly appreciated.

\section{References}

[1] R. Alferness, App1. Phys. 7 (1975) 29.

[2] R. Alferness and S.K. Case, J. Opt. Soc. Amer., in print.

[3] H. Kogelnik, Bell Syst. Tech. J. 48 (1969) 2909. 